\title{
Consensus development conference: prophylaxis and treatment of osteoporosis
}

\begin{abstract}
A consensus development conference sponsored by the European Foundation for Osteoporosis and Bone Disease was held at Aalborg from 3 to 4 October 1987. A panel of 13 listened to evidence from experts in public sessions attended by 700 people, including representatives of the medical profession, the pharmaceutical industry, the press, and ministries of health. After a closed session the panel discussed its report with the audience and a consensus statement was then presented at a press conference.
\end{abstract}

\section{Consensus statement}

Osteoporosis is a disorder characterised by a reduced amount of bony tissue per unit volume of bone. It is a major but not the sole cause of fractures in postmenopausal women and in the elderly.

Fractures occur most frequently at the wrist, the spine, and the hip with minimal trauma, and an osteoporotic woman may fracture any bone more easily than her non-osteoporotic counterpart.

Osteoporosis is an important health problem in the Western world. More than $40 \%$ of women will have experienced a fracture by the time they reach the age of 70 . Moreover, the incidence of hip fracture appears to be increasing, and this is explained only in part by a longer life expectancy.

Osteoporosis results in substantial costs and personal morbidity. Fractures cause pain and disability. Hip fractures are associated with a $5-20 \%$ excess mortality within the first year, and they often cause lasting functional disability. Each year 250000 patients are admitted to hospital in the United States with hip fracture, where its costs have been conservatively estimated to be at least $\$ 6$ billion a year. In some countries more hospital beds are occupied by patients with hip fractures than by patients with acute myocardial infarction. Research programmes on osteoporosis have now given us several possible approaches for both preventing postmenopausal bone loss and treating established osteoporosis. Since therapeutic attitudes vary widely throughout the world, this conference was devoted to prophylaxis and treatment of osteoporosis.

\section{(1) Prevention of bone loss by oestrogen/progestagen administration}

Oestrogen therapy prevents bone loss in postmenopausal women. It is currently the only well established prophylactic measure that reduces the frequency of osteoporotic fractures. The minimal effective dose of oral short acting oestrogen is $0.625 \mathrm{mg}$ of conjugated oestrogen, $2 \mathrm{mg}$ of $17 \beta$-oestradiol, or $25 \mu \mathrm{g}$ of ethinyloestradiol. Percutaneous $17 \beta$-oestradiol also seems to prevent postmenopausal bone loss, but no convincing data are available on transdermal $17 \beta$-oestradiol ("patch" oestrogen), and

The panel comprised: Drs Claude D Arnaud, Claus Christiansen, Steven $\mathbf{R}$ Cummings, Herbert Fleisch, Carlo Gennari, John A Kanis, John GG Ledingham, Iain MacIntyre, T Jack Martin, William A Peck, Povl Riis, Göran Samsioe; and Lawrence E Shulman.

Invited experts presenting evidence were: Drs Charles H Chesnut III, Barbara L Drinkwater, Ralph S Goldsmith, C Conrad Johnston Jr, Robert Lindsay, Thomas M Mack, Pierre J Meunier, B E Christopher Nordin, A Michael Parfitt, B Lawrence Riggs, John C Stevenson, and Malcolm I Whitehead. the optimal dose of percutaneous $17 \beta$-oestradiol remains to be defined.

Oestrogens have other effects, both favourable and unfavourable. Treatment with oestrogens alters the lipid and lipoprotein metabolism in a way that may favour prevention of atheroma. There is evidence that oestrogen therapy may reduce the incidence of cardiovascular disease. The most important potential effect of oestrogen may be to reduce the risk of coronary heart disease.

Oestrogens reduce or eliminate menopausal symptoms such as hot flushes, episodic sweating, vaginal dryness, and urethral irritation.

Oestrogen therapy is not uncomplicated. Both oral and to a lesser degree percutaneous administration of oestrogens stimulate hepatic protein synthesis, possibly increasing circulating concentrations of coagulation factors and renin substrate. Whether these changes are accompanied by increases in morbidity is uncertain. It is well documented that the risk of endometrial hyperplasia and cancer increases during and after oestrogen monotherapy. The risk is related to the dose and the duration of treatment. Well designed epidemiological studies do not suggest an overall increase in the risk of breast cancer in postmenopausal women treated with oestrogen.

Cyclical or continuous administration of sufficient amounts of progestogens controls vaginal bleeding, eliminates endometrial hyperplasia, and reduces the risk of endometrial cancer. Progestogens may have variable effects on serum lipids and lipoproteins and therefore might negate the possible beneficial cardiovascular effects of oestrogen. Further research is needed to evaluate the lipoprotein effects of different progestogens or schedules. The available evidence indicates that postmenopausal women identified to be at risk of developing osteoporosis should receive oestrogen therapy, provided there are no contraindications and careful follow up is ensured. Pending further information, the concomitant use of cyclic or continuous progestogen therapy is recommended in a woman with an intact uterus in order to control bleeding and to reduce the risk of endometrial cancer. Treatment should be started as soon after menopause as possible. The benefits of oestrogen therapy are greatest just after the menopause, and intervention 15 to 20 years later is not desirable. The appropriate duration of oestrogen treatment is unknown, but at least 10 years seems to be reasonable.

\section{(2) Calcium in the prevention of osteoporosis}

Nutritional intake of elemental calcium is an absolute requirement for bone health, facilitating growth and consolidation and reducing bone loss after skeletal maturity has been reached. The threshold of calcium intake below which bone health is jeopardised varies at different stages of life. Estimates of calcium requirements for achieving and maintaining bone mass have yielded conflicting results, but a daily nutritional requirement of $800 \mathrm{mg}$ is recommended for Europid women, with higher requirements likely in childhood, in adolescence, and during pregnancy and lactation.

Calcium and peak bone mass-The amount of bone achieved at maturity (peak bone mass) is determined by genetic and environmental factors. Evidence from several studies suggests that dietary calcium intake in childhood and adolescence may be a determinant of peak bone mass. It has yet to be shown that decreasing the intake of calcium in childhood and adolescence results in a decreased peak 
bone mass. It seems likely that an adequate calcium intake is necessary for the maintenance of peak bone mass, but it is not known whether a high calcium intake at this stage of adulthood can contribute to improvements in bone mass. There is a need for studies to address the question of the role of calcium supplementation in the third to fifth decades in women.

Calcium and bone loss-Calcium in the diet does not substitute for oestrogen in preventing perimenopausal bone loss. Some randomised clinical trials in postmenopausal women have found that a high calcium intake reduces the rate of loss of cortical but not of trabecular bone. Its efficacy in preventing fracture is uncertain. In postmenopausal women with established osteoporosis and women recognised to be at high risk of fracture it seems prudent for physicians to recommend a calcium intake of about $1500 \mathrm{mg}$ a day.

\section{(3) Exercise}

Acute immobilisation leads to loss of bone, which can be at least partially reversed by resumption of weight bearing activity. There is sufficient evidence to support the view that women should be encouraged to keep up a reasonable level of physical activity. Excessive exercise can lead to amenorrhoea and loss of bone. This very fact calls attention to the overriding importance of oestrogen in maintaining bone mass. The part played by exercise in reducing bone loss in the elderly is uncertain. It can be recommended in moderation, at least with the aim of improving patients' agility and reducing the likelihood of falls leading to fracture.

\section{(4) Fluoride}

Fluoride may be used to increase trabecular bone mass in patients with severe vertebral osteoporosis. It is the only agent that has been shown to have a sustained effect on the formation of trabecular bone both at appendicular and at axial sites.

Fluoride should not be used in patients with predominantly cortical osteoporosis; nor does it have a place in the prophylaxis of bone loss. Its use should be restricted to centres with skill in skeletal metabolism. There are several concerns and uncertainties regarding its widespread use.

Fluoride increases trabecular bone mass, but some studies have shown directly or indirectly a decrease in cortical bone mass. Recent anecdotal reports of hip fracture associated with fluoride treatment have emphasised this concern. It is prudent to reserve the use of fluoride for patients with severe vertebral osteoporosis. Whether treatment with fluoride reduces the rate of vertebral fracture is not known. Prospective controlled studies now in place should help clarify this issue.

Up to one third of patients do not respond to treatment with fluoride for reasons that are not yet known. In addition there are reversible side effects-including gastrointestinal intolerance, bone pain, and arthralgias - in a substantial minority of patients sufficient to necessitate stopping treatment. In the light of these side effects the overall risk-benefits ratio of fluoride treatment is uncertain.

There are some uncertainties about the optimum dose and duration of treatment. A view consistent with general experience suggests that doses of elemental fluoride of approximately $20 \mathrm{mg}$ daily are effective but may cause defective mineralisation, which can be overcome by the concurrent administration of calcium supplements with or without vitamin $\mathrm{D}$. The optimum dose and duration of fluoride treatment are not known, but the duration should probably not exceed five years.

\section{(5) Vitamin D and its metabolites and analogues}

Other than in the treatment of coexisting vitamin $\mathrm{D}$ deficiency the use of vitamin $\mathrm{D}$ derivatives is not recommended in postmenopausal osteoporosis. Vitamin D, its analogues, and its metabolites have been assessed both in the prevention of bone loss and in the treatment of established osteoporosis. In the early postmenopausal period there is no evidence that vitamin $D$, its analogues, or its metabolites decrease the rate of bone loss or fracture. There are conflicting and unresolved observations concerning the effect of vitamin $\mathrm{D}$ metabolites in decreasing the rate of bone loss in patients with established osteoporosis.

\section{Anabolic steroids}

Currently available anabolic steroids do not have a place in preventing osteoporosis in women. Although they can increase bone mass in women with established osteoporosis, perhaps by increasing bone formation, serious concerns remain about their safety. Potential side effects that limit their use include virilisation, a potentially atherogenic effect on plasma lipoproteins, liver dysfunction, and the possible development of liver tumours. Androgens do have a place in preventing and treating osteoporosis in hypogonadal males. The use of anabolic steroids in certain subsets of the osteoporotic population (for example, patients with glucocorticoid mediated osteoporosis) deserves further scrutiny.

\section{(7) Calcitonin}

The use of calcitonin may be considered in the primary prevention of osteoporosis in women at high risk who are not candidates for oestrogen therapy, although its efficacy in this context has not been extensively studied. Calcitonin may be effective in reducing subsequent bone loss in patients with established osteoporosis, but there is no substantive evidence that it reduces the frequency of fractures.

Although minor side effects are not uncommon, treatment with calcitonin is virtually bereft of major complications. Apart from the high cost its current use is limited by the necessity for parenteral administration. Intranasal formulations are now being tested. Long term studies are needed to determine whether patients with osteoporosis will become resistant to the effects of calcitonin.

\section{(8) Cyclic and coherence therapies}

Cyclic and coherence therapies represent promising experimental approaches to the prevention and treatment of osteoporosis. Cyclic therapy includes intermittent treatment with an inhibitor of bone resorption such as one of the diphosphonates or calcitonin. Less well studied is the intermittent use of stimulators of bone formation such as parathyroid hormone or sodium fluoride. In coherence therapy patients are exposed continuously or discontinuously to an activator of bone remodelling. Preliminary evidence from short term studies indicates that these regimens may reduce bone loss. Their safety and their effects on bone mass in the long term and on the frequency of fractures remain to be determined in randomised trials.

\section{Future research}

There is an urgent need for additional knowledge about the prevention and treatment of osteoporosis. It is imperative that research be directed towards:

(a) delineating the factors that determine the accumulation of bone tissue during growth and maturation, the losses of bone tissue thereafter, and the strength of the adult skeleton;

(b) identifying individuals at special risk for developing osteoporosis and fracture;

(c) developing accurate, precise, and inexpensive methods to monitor the effects of treatment; and

(d) generating preventive and treatment strategies that are safe, effective, and inexpensive and have a high degree of patient acceptance.

These advances will require improved understanding of basic bone biology and of the epidemiology of osteoporosis as well as randomised clinical trials of new treatment modalities. 